\title{
Correspondence
}

\section{Deadly dowry: how engulfment pathways promote cell killing}

\author{
Cell Death and Differentiation (2016) 23, 553-554; doi:10.1038/cdd.2015.170; published online 12 February 2016
}

\section{Dear Editor,}

During the development of the nematode Caenorhabditis elegans cell death occurs in a highly reproducible manner, and this is one of the reasons why the 'worm' has been a prime model for studies of this fundamental process. ${ }^{1}$ Genetic analyses of $C$. elegans provided the first evidence that a conserved central pathway of cell death exists and resulted in the discovery of Apaf-1-like proteins and caspases. ${ }^{2-4}$ Subsequent studies led to the model that cells programmed to die during $C$. elegans development undergo transcriptional upregulation of the $\mathrm{BH} 3$-only gene egl-1, and this leads to the cell-autonomous killing of these cells via the central apoptosis pathway. ${ }^{1}$ Once dead, 'cell corpses' are engulfed by neighboring cells in a process that is mediated by two partiallyredundant parallel pathways that are conserved as well. ${ }^{5}$

In two landmark papers published back-to-back in Nature in 2001, Hengartner and co-workers and Horvitz and co-workers presented evidence that the engulfment pathways do not simply act in a linear and sequential manner. ${ }^{6,7}$ Instead, they demonstrated that the engulfing cells use the engulfment pathways to actively promote the killing of cells programmed to die. They reported that when the apoptosis pathway is compromised (e.g., by partial inactivation of the ced-3 caspase gene) the inactivation of any of the engulfment genes significantly increases the number of inappropriately surviving, 'undead' cells. Furthermore, this 'killing function' of the engulfment genes is independent of the gene ced-8.

What is ced-8? The ced-8 gene encodes a putative Xkr8like phospholipid scramblase that, once cleaved by active CED-3 caspase in a dying cell, induces the externalization of phosphatidylserine (PtdSer) to the cell surface of this cell. ${ }^{8-10}$ On the cell surface PtdSer acts as an 'eat-me' signal that is recognized by the phagocytic receptor CED-1 mEGF10 expressed by neighboring cells. ${ }^{11}$ CED-1 mEGF10 receptor molecules subsequently become enriched at the region of contact, leading to the activation of the two engulfment pathways in the engulfing cell, followed by engulfment and degradation of the cell corpse. The observation that the 'killing function' of the engulfment pathways is independent of ced-8 strongly suggested that the engulfment pathways do more than simply promote engulfment. However, the molecular nature of this 'killing function' has remained enigmatic until now.

In a recent paper in Nature Communications, we and co-workers presented evidence that, indeed, it is a novel signaling function of the engulfment pathways that promotes the killing of cells programmed to die during $C$. elegans development. ${ }^{12}$ Interestingly, this novel signaling function involves not the cell that is programmed to die, but rather its mother. This is in line with the observation that most cells programmed to die during $C$. elegans development are generated through an asymmetric division. For example, the neurosecretory motor neuron (NSM) neuroblast divides asymmetrically to generate a larger cell, the NSM, and a smaller cell, the NSM sister cell. The NSM survives and differentiates into a serotonergic neuron, while its smaller sister undergoes apoptosis. When the asymmetry of the NSM neuroblast division is eliminated, not only are the NSM and the NSM sister cell of similar sizes, but the snail-like $\mathrm{Zn}$ finger transcription factor CES-1, which is normally only detectable in the larger NSM, is now segregated into both daughters. Since CES-1 is a transcriptional repressor of the egl-1 $\mathrm{BH} 3$-only gene, both daughters of the NSM neuroblast now survive. ${ }^{13}$

New tools that we recently developed allowed us to monitor both the level and the activity of CED-3 caspase within the NSM lineage. The first observation that these tools allowed us to make is that active CED-3 caspase is already present in the NSM neuroblast. Furthermore, we present evidence that at the time the NSM neuroblast is about to divide (at metaphase), CED-3 activity exists in a gradient within the cell. More CED-3 activity is present in the dorsal part of the NSM neuroblast, (inherited by the NSM sister cell), and less CED-3 is present in the ventral part (inherited by the NSM) (Figure 1). Based on this, we propose that this gradient results in the preferential segregation of active CED-3 caspase into the NSM sister cell, which is programmed to die. Furthermore, we also found that after NSM neuroblast division, there is an asymmetry between the two daughters with respect to the synthesis and/or stability of CED-3 protein. CED-3 protein levels progressively increase within the smaller NSM sister cell, but decrease in the larger NSM.

Having these two assays in hands, we then found that CED-3 caspase activity in the NSM neuroblast is dependent on the function of the entire central apoptosis pathway (egl-1 BH3-only, ced-9 Bcl-2, ced-4 Apaf-1 and ced-3 caspase), and that the establishment of the gradient of CED-3 caspase activity within the NSM neuroblast is dependent (at least in part) on components of both engulfment pathways. So, does the phagocytic receptor CED-1 mEGF10 accumulate around the NSM neuroblast? Indeed it does, but only on plasma membranes of the two dorsal neighboring cells (Figure 1). Furthermore, this accumulation is dependent on active CED-3 caspase. To test whether CED-3 caspase induces CED-1 mEGF10 accumulation around the dorsal 

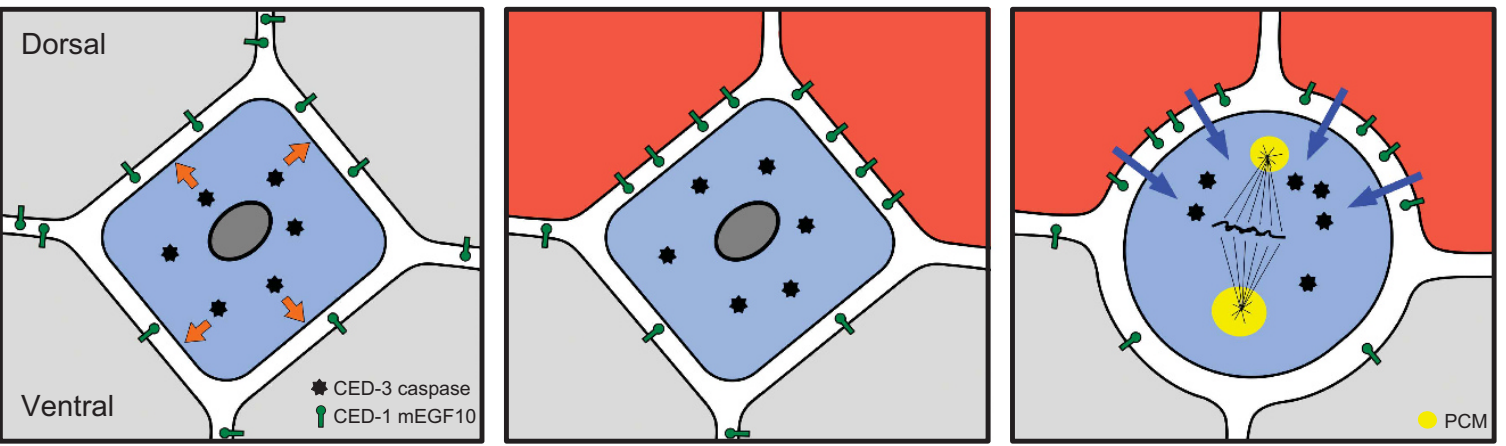

Figure 1 The engulfment pathways promote the killing of the NSM sister cell by contributing to the polarization of the NSM neuroblast, which is required for the unequal segregation of apoptotic potential. Left: Active CED-3 caspase present in the NSM neuroblast generates a 'polarize-me signal' (orange arrows). Middle: The 'polarize-me signal' induces the accumulation of the phagocytic receptor CED-1 mEGF10 on plasma membranes of neighbors that face the dorsal side of the NSM neuroblast. This accumulation leads to the activation of the two partially-redundant, parallel engulfment pathways in the two dorsal neighbors (indicated by orange shading). Right: NSM neuroblast at metaphase. The activities of the engulfment pathways in the dorsal neighbors generate a signal (blue arrows) that contributes to the polarization of the NSM neuroblast along the dorsal-ventral axis. This dorsal-ventral polarity is reflected in the gradient of active CED-3 caspase that is established and the differing amounts of peri-centriolar material (PCM) that is associated with the two centrosomes. As a result of this polarity 'apoptotic potential' (including active CED-3 caspase) is unequally segregated during NSM neuroblast division with the daughter that inherits the dorsal part of the NSM neuroblast (the NSM sister cell) inheriting more of apoptotic potential than the daughter that is formed from the ventral part (the NSM). See text for details

side of the NSM neuroblast by activating CED-8 Xkr8 and PtdSer externalization, we then analyzed ced-8 mutants and found that the loss of ced-8 had no effect. Evidently, a signal other than PtdSer triggers CED-1 mEGF10 accumulation and the activation of the two engulfment pathways. Finally, not only is the gradient of CED-3 caspase activity in the NSM neuroblast disrupted in ced-1 mEGF10 mutants, but so was the ability of the NSM sister cell to synthesize additional CED-3 protein. Based on this we propose that both active CED-3 caspase itself and the potential to synthesize additional CED-3 protein are present within a gradient in the NSM neuroblast at metaphase. The unequal segregation of 'apoptotic potential' would explain one conundrum of cell death during $C$. elegans development, which is the speed with which it occurs. About $\sim 30$ min after being generated, cells programmed to die, such as the NSM sister cell, already exhibit morphological and molecular characteristics of apoptotic cells and within another $\sim 30 \mathrm{~min}$, their corpses are completely digested by neighboring cells.

Many questions remain. For example, we do not know how CED-1 mEGF10 accumulation around the NSM neuroblast is induced and how, in turn, the engulfment pathways affect the polarization of the NSM neuroblast. And, it is unknown how the synthesis/stability of CED-3 protein is controlled in the NSM and NSM sister cell, and how the central apoptosis pathway is induced and regulated in the NSM neuroblast in the first place. Furthermore, how relevant might this be to higher organisms? It is well known that professional phagocytes (particularly macrophages) can contribute to cell killing in certain contexts in vitro and in vivo. ${ }^{14,15}$ However, in these cases the 'killing function' of the phagocytes has been ascribed to their ability to engulf the doomed cells. Whether the conserved engulfment pathways can promote cell killing by promoting the segregation of apoptotic potential during cell division in higher organisms remains to be determined.

\section{Conflict of Interest}

The authors declare no conflict of interest.

\section{EJ Lambie ${ }^{1}$ and B Conradt ${ }^{*, 1}$}

${ }^{1}$ Department of Biology II, Ludwig-Maximilians-University of Munich, Center for Integrated Protein Science Munich-CIPS ${ }^{\mathrm{M}}$, LMU Biocenter, PlaneggMartinsried, Germany

*Corresponding authors: B Conradt, Department of Biology II, LudwigMaximilians-Universität München (LMU), Center for Integrated Protein Science Munich-CIPS ${ }^{\mathrm{M}}$, LMU Biocenter, Großhaderner Straße 2, Planegg-Martinsried 82152, Germany. Tel: +49 (0)89 2180 74217; Fax: +49 (0)89 2180 74219;

E-mail: conradt@bio.Imu.de

1. Horvitz HR. Biosci Rep 2003; 23: 239-303.

2. Yuan J, Horvitz HR. Development 1992; 116: 309-320.

3. Yuan J et al. Cell 1993; 75: 641-652.

4. Ellis HM, Horvitz HR. Cell 1986; 44: 817-829.

5. Mangahas PM, Zhou Z. Semin Cell Dev Biol 2005; 16: 295-306.

6. Hoeppner DJ et al. Nature 2001; 412: 202-206.

7. Reddien PW et al. Nature 2001; 412: 198-202.

8. Chen YZ et al. Nat Commun 2013; 4: 2726.

9. Stanfield GM, Horvitz HR. Mol Cell 2000; 5: 423-433.

10. Suzuki J et al. Science 2013; 341: 403-406.

11. Li Z et al. PLoS Genet 2015; 11: e1005285.

12. Chakraborty $S$ et al. Nat Commun 2015; 6: 10126.

13. Hatzold J, Conradt B. PLoS Biol 2008; 6: e84.

14. Conradt B. Nat Cell Biol 2002; 4: E139-E143.

15. Mallat M et al. Curr Opin Neurobiol 2005; 15: 101-107.

(1)(2) This work is licensed under a Creative Commons cc) Attribution-NonCommercial-ShareAlike 4.0 International License. The images or other third party material in this article are included in the article's Creative Commons license, unless indicated otherwise in the credit line; if the material is not included under the Creative Commons license, users will need to obtain permission from the license holder to reproduce the material. To view a copy of this license, visit http://creativecommons.org/licenses/by-nc-sa/4.0/ 